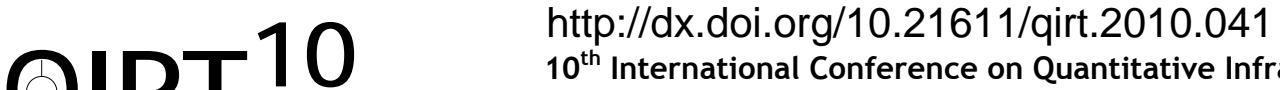 \\ $10^{\text {th }}$ International Conference on Quantitative InfraRed Thermography \\ July 27-30, 2010, Québec (Canada)
}

\section{Application of tele- and thermovision to assessment of quality of welded joints}

\author{
by M. Fidali*, A. Timofiejczuk*, A. Bzymek*, W. Jamrozik* A. Czupryński** \\ * Dept. of Fundamentals of Machinery Design, Silesian University of Technology, Poland, Gliwice, \\ Konarskiego 18a str., marek.fidali@polsl.pl \\ **Welding Dept., Silesian University of Technology, Poland, Gliwice, Konarskiego 18a str., artur.czuprynski@polsl.pl
}

\begin{abstract}
High quality welded joints are often an effect of constant control of welding process parameters. However, during production the assurance of correct parameters of the process does not always follow a defect free joint. A crucial problem in the assessment of the quality of joints is to detect subsurface defects which are undetectable with the use of a visual inspection. Detection of hidden defects of welded joints is commonly performed by the application of different destructive and non-destructive tests such as metallographic research, radiography, ultrasonic etc. These methods are very efficient but expensive and time consuming. Additionally, they do not allow us to check each single joint of a produced part.

More often and often the maintenance of the welding process is performed on-line by means of monitoring of process parameters [2]. An alternative and most promising way of the maintenance of these processes is to employ a visual control $[1,3,4,6]$. The process can be observed both in infrared and visible electromagnetic bands. Some solutions are teleand/or thermovision observations. They make possible to identify abnormalities occurring during the process rapidly. These abnormalities often affect the joint quality. It is important that in order to perform an effective on-line vision monitoring of the welding process certain image processing and analysis methods should be applied.

Presented research has been devoted to the detection of different kinds of welded joint defects. A multimodal data processing was decided to be applied. The novel approach has proposed for data processing and analysis is the application of image and data fusion methods which emphasize such features of images and signals which are unidentifiable through their separate analyses. The article has been focused on a part of the research dedicated to the processing, analysis and fusion of TV and IR images. In order to acquire welding data suitable for assessing of quality of welded joints the observation of the process was performed with the use of a monitoring system consisting of three cameras (two TVs and one IR) as well as voltage, current and sound transducers. Positions of the cameras are shown in Fig 1.
\end{abstract}

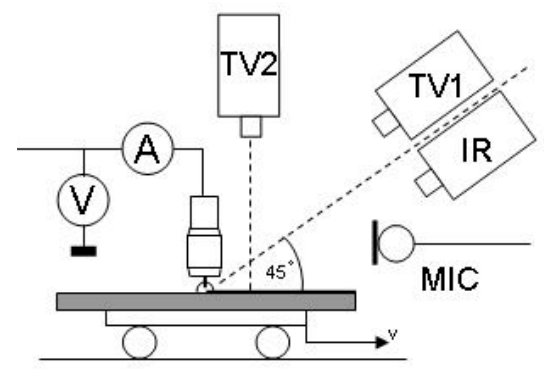

Fig. 1. Positions of sensors observing the welding process.

The IR and TV1 cameras observing a welding arc, pool, and joint that is getting cold. The second TV2 camera observed the welded joint. In order to improve the quality of the images acquired by means of the TV2 camera, the observed scene was illuminated. The images as well as additional process signals were acquired synchronously with the use of the software developed in LabView.

There were two goals of the image analysis. Firstly, we assessed the stability of the welding process which was performed by means of estimation geometrical parameters of the arc. Secondly, some common defects of joints were possible to be detected. The processing of images recorded during the observation of the process was divided into several steps. At first, a few regions of interest (ROIs) were selected. One decided to distinguish ROIs representing the welding arc and joint (IR camera), welding arc (TV1 camera) and joint (TV2 camera) respectively. The processing procedures, applied to ROIs deriving from images recorded by TV cameras as well as IR camera, were similar and consisted in normalization, filtration and binarization. The main difference between these procedures were some input parameters, especially values of a binarization threshold. These values were subjects of very detailed experiments.

As opposed to processing, three types of recorded images, required different approaches to their analysis. These operations were limited to distinguished ROIs only. The goal of the analysis was to obtain series of image features that could be the 
background for the recognition of abnormalities of the welding process and defects of the joint. It was assumed that a set of selected relevant features provided us with information enabling image classification into one of classes defined a priori.

The analysis of processed ROIs of thermal and vision images could be carried out with the use of numerous methods [1,3,6].

Stability of the welding process was estimated on the basis of images of the welding arc. In this case numerous statistical as well as topological features could be estimated. The result of the methods was a huge set of features. To select the relevant ones commonly known feature selection methods e.g. correlation analysis were applied. The observation of changes of selected features let us estimate abnormalities of the process. Among other factors, the stability of the welding arc was dependent on the quality of elements being welded as well as the welding parameters. In case of correctly prepared edges of welded elements the process was stable. Small deviations of the arc and shape dimensions were only observed. To classify the abnormalities of the process, certain pattern shapes of the arc were determined.

Procedures elaborated for images representing welded joints were based on manipulation and analysis of image brightness and temperature profiles along and across the welded joint. The profiles could be calculated along straight lines which were perpendicular and parallel to the main axes of the weld. Such profiles could be treated as specific functions and analyzed with the use of statistics known from impulse and transient signal estimation. Ordering the profiles according to the length or width of the welded joint let us acquire a set of profiles called profilograms or scannograms. Such set could be represented also in a form of images and analyzed. Scannograms enabled us to identify the width of the weld face and estimate irregularities of its surface and edges. Employment of the scannograms made it also possible to identify such welding faults as concavity of the weld, metal spattering, partial or lack of penetration.

TV and IR images can be also represented in different forms. Examples are Fourier images obtained as results of Fourier transform. Afterwards, such new images could be analyzed with the use of above mentioned methods as well as with especially dedicated estimators. In order to estimate F-images of considered images such spectral features as Longitual Fourier Power (LFP), Transversal Fourier Power (TFP), or Circular Fourier Power (CFP) were proposed and estimated. The first two features were values of the Fourier spectrum calculated respectively for horizontal and vertical areas with reference to image axes. CFP parameter was estimated as the mean Fourier power calculated along a circle.

Images acquired by means of IR and TVI cameras presented the common part of the same scene and could be analysed jointly after their fusion, which consisted in aggregation of several images in order to obtain a new synthetic image that was more informative than each of individual images acquired [7]. Images gathered by different devices had different characteristics and provided different, sometimes complementary information. According to that the application of techniques of image fusion made it possible to perceive features which were impossible to be discovered when corresponding single images were analyzed.

The fusion was preceded with a registration. The operation was a process of overlapping of two or more images. Typically, the registration consist in the calculation of some parameters of geometrical transformation. The proposed registration was based on the spectral analysis and Fourier - Mellin transform. Certain basic geometrical parameters such as translation in two axes, rotation and scale were calculated for each image pair.

During the preliminary research several fusion algorithms were considered, e.g. various hierarchical (multiresolution) approaches, nonlinear methods incorporating min, max, and mean operators as well as methods based on probabilistic and evidence theory. Since the fusion algorithm was dependent on the application, the nonlinear and multiresolution (including wavelet) methods were applied. They were relatively easy to be implemented and flexible enough to deal with the assessment of the welding process stability. The methods appeared to be sensitive to local contrast changes, e.g. the edges or corners. To enhance certain features that let us reveal instabilities of the welding process for each fusion method special rules were elaborated. In the proposed approach the fusion was performed on the low-frequency / low-band levels and the rule preferring maximal values or the weighted average rule (weights were determined individually for each multiresolution decomposition method) were employed.

\section{REFERENCES}

[1] Bzymek A., Fidali M., Timofiejczuk A.: Methods of image processing in vision system for assessing welded joints quality. Recent Advances in Mechatronics. Jabłoński R., Turkowski M., Szewczyk R. (ed.), Springer,258-262, 2007.

[2] Fan H., Ravala N.K., W ikle H.C., Chin B.A.: Low-cost infrared sensing system for monitoring the welding process in the presence of plate inclination angle. J ournal of Materials Processing Technology vol. 140, no 1: 668-675, 2003.

[3] Fidali M., Timofiejczuk A.: Assessment of welded joints quality by means of analysis of its thermovision images recorded during welding process. Diagnostyka, 1(49), 2009, p. 49-56

[4] Huang R.-S., Liu L.-M., Song G. : Infrared temperature measurement and interference analysis of magnesium alloys in hybrid laser-TIG welding process. Mater. Sci. Eng. A, 2006, doi:10.1016/j.msea.2006.10.069.

[5] Węglowski M. S.: Visible radiation as an useful signal in monitoring of welding process. Pomiary Automatyka Robotyka 10/2006 (In Polish)

[6] Xu D., Wang L., Tan M.: Image processing and visual control method for arc welding robot, Proceedings of the IEEE International Conference on Robotics and Biomechanics, Shenyang, China 2004

[7] Zitová B., Flusser J .: Image registration methods: a survey. Image Vision Comput. 21(11): pp. 977-1000, 2003 